\title{
AN ANALYTICAL SOLUTION OF THE WEIGHTED FERMAT-TORRICELLI PROBLEM ON THE UNIT SPHERE
}

\author{
ANASTASIOS N. ZACHOS
}

\begin{abstract}
We obtain an analytical solution for the weighted FermatTorricelli problem for an equilateral geodesic triangle $\triangle A_{1} A_{2} A_{3}$ which is composed by three equal geodesic arcs (sides) of length $\frac{\pi}{2}$ for given three positive unequal weights that correspond to the three vertices on a unit sphere. This analytical solution is a generalization of Cockayne's solution given in 4 for three equal weights. Furthermore, by applying the geometric plasticity principle and the spherical cosine law, we derive a necessary condition for the weighted Fermat-Torricelli point in the form of three transcedental equations with respect to the length of the geodesic arcs $A_{1} A_{1}^{\prime}$, $A_{2} A_{2}^{\prime}$ and $A_{3} A_{3}^{\prime}$, to locate the weighted Fermat-Torricelli point $A_{0}$ at the interior of a geodesic triangle $\triangle A_{1}^{\prime} A_{2}^{\prime} A_{3}^{\prime}$ on a unit sphere with sides less than $\frac{\pi}{2}$.
\end{abstract}

\section{INTRODUCTION}

Let $\triangle A_{1} A_{2} A_{3}$ be a geodesic triangle and $A_{0}$ a point on a unit sphere.

We denote by $a_{i j}$ the length of the geodesic arc $A_{i} A_{j}$, which is part of a great circle of unit radius and $\alpha_{i k j}$ the angle between the geodesic $\operatorname{arcs} A_{i} A_{k}$ and $A_{k} A_{j}$ for $i, j, k=0,1,2,3, i \neq j \neq k$.

The weighted Fermat problem on the unit sphere refers to the following problem:

Problem 1. Consider a positive constant weight $w_{i}$ that correspond to the vertex $A_{i}$, for $i=1,2,3$. Find a point $A_{0}$ (weighted Fermat point) for which the sum

$$
\sum_{i=1}^{3} w_{i} a_{0 i}
$$

is minimized.

1991 Mathematics Subject Classification. 52A40,51E10,51M16,52A55.

Key words and phrases. Fermat-Torricelli problem, spherical triangle. 
The existence and uniqueness for the weighted Fermat point on a convex surface has been studied in [10], [6], [2], [11] (see also in [7] [1, Chapter II,pp. 208]).

Concerning some studies that focus on the geometric properties of the weighted Fermat point on the two dimensional sphere and on a convex surface we refer to the studies of [4], [5], [9], [3], [7], [8] and [13].

The following results (Proposition 1,2) characterize the solutions of the weighted Fermat problem on a $C^{2}$ surface and they have been proved in [11],2] proposition 6 , page 53 and proposition 7 , page 55:

Proposition 1 (Floating Case). [11, Proposition 6, p. 53], [2] If $\vec{U}_{A_{i} A_{j}}$ is the unit tangent vector of the geodesic arc $A_{i} A_{j}$ at $A_{i}$ and $D$ is the domain of a $C^{2}$ surface $M$ bounded by $\triangle A_{1} A_{2} A_{3}$, for $i, j=1,2,3$ then the following (I), (II), (III) conditions are equivalent: (I) All the following inequalities are satisfied simultaneously:

$$
\begin{aligned}
& \left\|w_{2} \vec{U}_{A_{1} A_{2}}+w_{3} \vec{U}_{A_{1} A_{3}}\right\|>w_{1}, \\
& \left\|w_{1} \vec{U}_{A_{2} A_{1}}+w_{3} \vec{U}_{A_{2} A_{3}}\right\|>w_{2}, \\
& \left\|w_{1} \vec{U}_{A_{3} A_{1}}+w_{2} \vec{U}_{A_{3} A_{2}}\right\|>w_{3},
\end{aligned}
$$

(II) The point $A_{0}$ is an interior point of $\triangle A_{1} A_{2} A_{3}$ (weighted FermatTorricelli point) and does not belong to the geodesic arcs $A_{1} A_{2}, A_{2} A_{3}$ and $A_{1} A_{3}$.

$$
\text { (III) } \vec{U}_{A_{0} A_{1}}+\vec{U}_{A_{0} A_{2}}+\vec{U}_{A_{0} A_{3}}=\overrightarrow{0} \text {. }
$$

Proposition 2 (Absorbed Case). [11, Proposition 7, p. 55], [2] The following (I), (II) conditions are equivalent.

(I) One of the following inequalities is satisfied:

$$
\left\|w_{2} \vec{U}_{A_{1} A_{2}}+w_{3} \vec{U}_{A_{1} A_{3}}\right\| \leq w_{1}
$$

or

$$
\left\|w_{1} \vec{U}_{A_{2} A_{1}}+w_{A_{3}} \vec{U}_{A_{2} A_{3}}\right\| \leq w_{2}
$$

or

$$
\left\|w_{1} \vec{U}_{A_{3} A_{1}}+w_{B} \vec{U}_{A_{3} A_{2}}\right\| \leq w_{3} .
$$

(II) The point $A_{0}$ (weighted Fermat-Cavalieri point) is attained at $A_{1}$ or $A_{2}$ or $A_{3}$, respectively. 
We note that there is no analytical solution with respect to the weighted Fermat-Torricelli problem on the unit sphere, except of Cockayne's solution given in [4] for an equilateral geodesic triangle having sides with length $\frac{p i}{2}$ for three equal weights.

In this paper, we find an analytical solution of the weighted FermatTorricelli problem for an equilateral geodesic triangle on a unit sphere which is composed by three equal geodesic arcs of length $\frac{\pi}{2}$, by using as variables the two angles of longitude and latitude from the spherical coordinates and by applying the spherical sine law in some specific geodesic triangles (Theorem 1). The geometric plasticity principle which has been proved in [13] yields a class of geodesic triangles such that the corresponding weighted Fermat-Torricelli point remains the same. By applying the geometric plasticity principle, we find a class of geodesic triangles by using the cosine law on the unit sphere, such that the weighted Fermat-Torricelli point is the same with the weighted Fermat-Torricelli point which corresponds to the equilateral geodesic triangle. Finally, by applying the geometric plasticity principle and the spherical cosine law, we derive a necessary condition for the weighted Fermat-Torricelli point in the form of three transcedental equations with respect to some specific three length of geodesic arcs to locate the weighted Fermat-Torricelli point $A_{0}$ at the interior of a geodesic triangle $\triangle A_{1}^{\prime} A_{2}^{\prime} A_{3}^{\prime}$ on a unit sphere with sides less than $\frac{\pi}{2}$ (Proposition 4).

\section{Analytical solution of the Weighted}

FERMAT-TORRICELLI PROBLEM ON THE UNIT SPHERE

Let $\triangle A_{1} A_{2} A_{3}$ be a geodesic triangle on the unit sphere $S: x^{2}+y^{2}+$ $z^{2}=1$. such that $a_{12}=a_{23}=a_{31}=\frac{\pi}{2}$ and $A_{1}=(1,0,0), A_{2}=(0,1,0)$, $A_{3}=(0,0,1)$.

Lemma 1. [10, Theorem 1], 12] If $A_{0}$ is the weighted Fermat-Torricelli point of $\triangle A_{1} A_{2} A_{3}$, then each angle $\alpha_{i 0 j}$ is expressed as a function of $w_{1}, w_{2}$ and $w_{3}$ :

$$
\alpha_{i 0 j}=\arccos \left(\frac{w_{k}^{2}-w_{i}^{2}-w_{j}^{2}}{2 w_{i} w_{j}}\right)
$$

for $i, j, k=1,2,3$, and $k \neq i \neq j$.

We start by expressing the position of the weighted Fermat-Torricelli point $A_{0}=(x, y, z)$ in terms of the spherical coordinates $(\omega, \varphi)$ :

$$
A_{0}=(\cos \omega \cos \varphi, \cos \omega \sin \varphi, \sin \omega) \text {. }
$$

Theorem 1. The analytical solution of the weighted Fermat-Torricelli problem of $\triangle A_{1} A_{2} A_{3}$ on the unit sphere is given by the following two relations: 


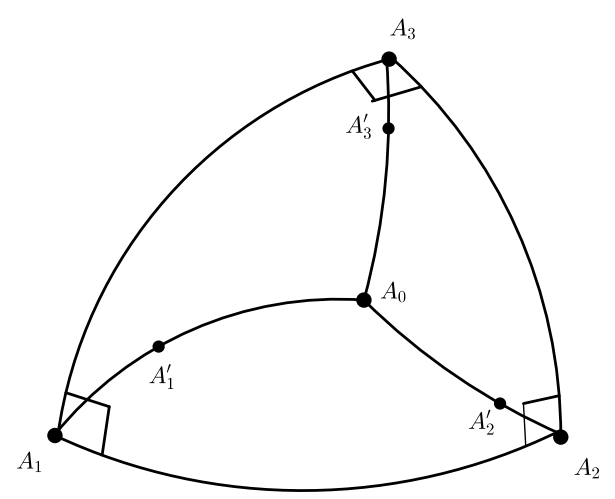

FIGURE 1. Location of the weighted Fermat-Torricelli point for a geodesic triangle on the unit sphere

$$
\varphi=\arccos \left(\sqrt{\frac{w_{1}^{2}+w_{3}^{2}-w_{2}^{2}}{2 w_{3}^{2}}}\right)
$$

and

$\omega=\arccos \left(\sqrt{\frac{w_{1}^{2}+w_{2}^{2}-w_{3}^{2}}{2 w_{1} w_{2} \sin \left(\arccos \left(\frac{w_{3}^{2}-w_{1}^{2}-w_{2}^{2}}{2 w_{1} w_{2}}\right)\right) \sin \left(\arccos \left(\frac{w_{2}^{2}-w_{1}^{2}-w_{3}^{2}}{2 w_{1} w_{3}}\right)\right)}}\right)$,

which yield the exact location of the weighted Fermat-Torricelli point $A_{0}$.

Proof. The location of $A_{0}=(\cos \omega \cos \varphi, \cos \omega \sin \varphi, \sin \omega)$. is determined by $\omega$ and $\varphi$.

We proceed by calculating $\omega$ and $\varphi$ with respect to the given positive weights $w_{1}, w_{2}$ and $w_{3}$.

By applying the sine law in $\triangle A_{1} A_{0} A_{3}, \triangle A_{1} A_{0} A_{2}$ and $\triangle A_{2} A_{0} A_{3}$, we get, respectively: 


$$
\begin{gathered}
\frac{1}{\sin \alpha_{103}}=\frac{\sin a_{03}}{\sin \alpha_{013}}=\frac{\sin a_{01}}{\sin \alpha_{130}} \\
\frac{1}{\sin \alpha_{102}}=\frac{\sin a_{02}}{\sin \left(\frac{\pi}{2}-\alpha_{013}\right)}=\frac{\sin a_{01}}{\sin \alpha_{120}}
\end{gathered}
$$

and

$$
\frac{1}{\sin \alpha_{203}}=\frac{\sin a_{03}}{\sin \left(\frac{\pi}{2}-\alpha_{120}\right)}=\frac{\sin a_{02}}{\sin \left(\frac{\pi}{2}-\alpha_{130}\right)} .
$$

By taking the orthogonal projection of $A_{0}$ with respect to the $x y$ plane and by using the Euclidean sine law, we express $a_{01}, a_{02}$ and $a_{03}$ as functions of $\omega$ and $\varphi$ :

$$
\begin{gathered}
\cos a_{03}=\sin \omega, \\
\cos a_{01}=\cos \omega \cos \varphi
\end{gathered}
$$

and

$$
\cos a_{02}=\cos \omega \sin \varphi
$$

By replacing (2.7), (2.8) and (2.9) in (2.4), (2.5) and (2.6) we obtain:

$$
\begin{gathered}
\frac{1}{\sin \alpha_{103}}=\frac{\cos \omega}{\sin \alpha_{013}}=\frac{\sqrt{1-\cos ^{2} \omega \cos ^{2} \varphi}}{\sin \alpha_{130}} \\
\frac{1}{\sin \alpha_{102}}=\frac{\sqrt{1-\cos ^{2} \omega \sin ^{2} \varphi}}{\cos \alpha_{013}}=\frac{\sqrt{1-\cos ^{2} \omega \cos ^{2} \varphi}}{\sin \alpha_{120}}
\end{gathered}
$$

and

$$
\frac{1}{\sin \alpha_{203}}=\frac{\cos \omega}{\cos \alpha_{120}}=\frac{\sqrt{1-\cos ^{2} \omega \sin ^{2} \varphi}}{\cos \alpha_{130}} .
$$

From (2.10) we get:

$$
\sin \alpha_{013}=\sin \alpha_{103} \cos \omega
$$

and

$$
\sin \alpha_{130}=\sin \alpha_{103} \sqrt{1-\cos ^{2} \omega \cos ^{2} \varphi} .
$$

From (2.11) we get:

$$
\cos \alpha_{013}=\sin \alpha_{102} \sqrt{1-\cos ^{2} \omega \sin ^{2} \varphi}
$$


and

$$
\sin \alpha_{120}=\sin \alpha_{102} \sqrt{1-\cos ^{2} \omega \cos ^{2} \varphi} .
$$

From (2.12) we get:

$$
\cos \alpha_{120}=\sin \alpha_{203} \cos \omega
$$

and

$$
\cos \alpha_{130}=\sin \alpha_{203} \sqrt{1-\cos ^{2} \omega \sin ^{2} \varphi} .
$$

By squaring both parts of (2.13) and (2.15) and by adding the two derived equations we obtain:

$$
\sin ^{2} \alpha_{103} \cos ^{2} \omega+\sin ^{2} \alpha_{102}\left(1-\cos ^{2} \omega \sin ^{2} \varphi\right)=1 .
$$

By squaring both parts of (2.14) and (2.18) and by adding the two derived equations we obtain:

$$
\sin ^{2} \alpha_{203} \cos ^{2} \omega+\sin ^{2} \alpha_{102}\left(1-\cos ^{2} \omega \cos ^{2} \varphi\right)=1 .
$$

By subtracting (2.20) from (2.19) and taking into account (2.1) from lemma 1 we derive:

$$
\cos 2 \varphi=\frac{B_{1}^{2}-B_{2}^{2}}{B_{3}^{2}}
$$

which yields (2.2).

By adding (2.20) from (2.19) and taking into account the trigonometric identity

$\sin ^{2} \alpha_{102}=\sin ^{2} \alpha_{203} \cos ^{2} \alpha_{103}+\cos ^{2} \alpha_{203} \sin ^{2} \alpha_{103}+2 \sin \alpha_{203} \cos \alpha_{203} \sin \alpha_{103} \cos \alpha_{103}$ and (2.1) from lemma 1 we derive (2.3).

Corollary 1. If $w_{1}=w_{2}=w_{3}$, then $\omega=\frac{\pi}{4}$ and $\varphi=\arccos \sqrt{\frac{2}{3}}$ and $A_{0}=\left(\frac{1}{\sqrt{3}}, \frac{1}{\sqrt{3}}, \frac{1}{\sqrt{3}}\right)$

Proof. By replacing $w_{1}=w_{2}=w_{3}$ in (2.2) and (2.3) we derive that $\omega=\frac{\pi}{4}$ and $\varphi=\arccos \sqrt{\frac{2}{3}}$ and we deduce the position of the FermatTorricelli point $A_{0}=\left(\frac{1}{\sqrt{3}}, \frac{1}{\sqrt{3}}, \frac{1}{\sqrt{3}}\right)$.

The geometric plasticity principle of quadrilaterals on a convex surface $M$, which is also valid on the unit sphere, states that ([13]): 
Proposition 3 (Geometric plasticity Principle). [13, Theorem 3, Proposition 8] Suppose that the weighted floating case of the weighted Fermat point $A_{0}$ point with respect to $A_{1} A_{2} A_{3} A_{4}$ is valid:

$$
\left\|w_{Q} \vec{U}_{R Q}+w_{S} \vec{U}_{R S}+w_{P} \vec{U}_{R P}\right\|>w_{R},
$$

for each $R, Q, S, P \in\left\{A_{1}, A_{2}, A_{3}, A_{4}\right\}$. If $A_{0}$ is connected with every vertex $R$ for $R \in\left\{A_{1}, A_{2}, A_{3}, A_{4}\right\}$ and we select a point $R^{\prime}$ with nonnegative weight $w_{R}$ which lies on the shortest arc $R A_{0}$ and the quadrilateral $A_{1}^{\prime} A_{2}^{\prime} A_{3}^{\prime} A_{4}^{\prime}$ is constructed such that:

$$
\left\|w_{Q} \vec{U}_{R^{\prime} Q^{\prime}}+w_{S} \vec{U}_{R^{\prime} S^{\prime}}+w_{P} \vec{U}_{R^{\prime} P^{\prime}}\right\|>w_{R^{\prime}},
$$

for $, R^{\prime}, Q^{\prime}, S^{\prime}, P^{\prime} \in\left\{A_{1}^{\prime}, A_{2}^{\prime}, A_{3}^{\prime}, A_{4}^{\prime}\right\}$. Then the weighted Fermat-Torricelli point $A_{0}^{\prime}$ is identical with $A_{0}$.

Lemma 2. The geometric plasticity principle holds for a geodesic triangle on the unit sphere.

Proof. By replacing $w_{4}=0$ in Proposition 3 , we deduce the geometric plasticity principle of a geodesic triangle $\triangle A_{1} A_{2} A_{3}$ on the unit sphere.

Let $\triangle A_{1}^{\prime} A_{2}^{\prime} A_{3}^{\prime}$ be a geodesic triangle on the unit sphere, such that $A_{i}^{\prime}$ belongs to the geodesic arc $A_{0} A_{i}$, for $i=1,2,3$, where $A_{0}$ is the weighted Fermat-Torricelli point of $\triangle A_{1} A_{2} A_{3}$ (Fig. 1).

We assume that $a_{12}^{\prime}, a_{23}^{\prime}, a_{31}^{\prime} \leq \frac{\pi}{2}$, in order to locate the geodesic triangle $\triangle A_{1}^{\prime} A_{2}^{\prime} A_{3}^{\prime}$ at the interior of $\triangle A_{1} A_{2} A_{3}$.

Furthermore, we assume that the same weight $w_{i}$ that corresponds to the vertex $A_{i}$ corresponds to the vertex $A_{i}^{\prime}$, for $i=1,2,3$, such that the inequalities of the weighted floating case hold (Proposition 1).

We denote by $A_{0}^{\prime}$ the corresponding weighted Fermat-Torricelli point of $\triangle A_{1}^{\prime} A_{2}^{\prime} A_{3}^{\prime}$.

We denote by $a$ the length of the geodesic arc $A_{1} A_{1}^{\prime}$, by $b$ the length of the geodesic arc $A_{2} A_{2}^{\prime}$ and by $c$ the length of the geodesic arc $A_{3} A_{3}^{\prime}$.

Proposition 4. The following system of three equations with respect to $a, b$ and $c$ provide a necessary condition to locate the weighted FermatTorricelli point $A_{0} \equiv A_{0}^{\prime}$ at the interior of a geodesic triangle $\triangle A_{1}^{\prime} A_{2}^{\prime} A_{3}^{\prime}$ on a unit sphere with sides less than $\frac{\pi}{2}$ :

$$
\cos a_{12}^{\prime}=\cos \left(a_{01}-a\right) \cos \left(a_{02}-b\right)+\sin \left(a_{01}-a\right) \sin \left(a_{02}-b\right) \frac{w_{3}^{2}-w_{1}^{2}-w_{2}^{2}}{2 w_{1} w_{2}}
$$




$$
\begin{aligned}
& \cos a_{23}^{\prime}=\cos \left(a_{02}-b\right) \cos \left(a_{03}-c\right)+\sin \left(a_{02}-b\right) \sin \left(a_{03}-c\right) \frac{w_{1}^{2}-w_{2}^{2}-w_{3}^{2}}{2 w_{2} w_{3}}, \\
& \text { and }
\end{aligned}
$$

$$
\cos a_{13}^{\prime}=\cos \left(a_{01}-a\right) \cos \left(a_{03}-c\right)+\sin \left(a_{01}-a\right) \sin \left(a_{03}-c\right) \frac{w_{2}^{2}-w_{1}^{2}-w_{3}^{2}}{2 w_{1} w_{3}}
$$

Proof. From lemma 2, the geometric plasticity holds on the units sphere. Therefore, $A_{0}=A_{0}^{\prime}$. By applying the cosine law in $\triangle A_{1}^{\prime} A_{0} A_{2}^{\prime}, \triangle A_{2}^{\prime} A_{0} A_{3}^{\prime}$ and $\triangle A_{1}^{\prime} A_{0} A_{3}^{\prime}$, we obtain (2.22), (2.23) and (2.24), respectively. The equations (2.22), (2.23) and (2.24) yield a system of three equations with respect to $a, b$ and $c$, because $a_{01}, a_{02}$ and $a_{03}$ could be expressed explicitly as functions of $w_{1}, w_{2}$ and $w_{3}$ taking into consideration the exact location of $\triangle A_{1} A_{2} A_{3}$ which has been given in Theorem 1 .

Remark 1. By replacing the Weirstrass transformations $\sin a=\frac{2 t_{a}}{1+t_{a}^{2}}$, $\cos a=\frac{1-t_{a}^{2}}{1+t_{a}^{2}}, \sin b=\frac{2 t_{b}}{1+t_{b}^{2}}, \cos b=\frac{1-t_{b}^{2}}{1+t_{b}^{2}}, \sin c=\frac{2 t_{c}}{1+t_{c}^{2}}, \cos c=\frac{1-t_{c}^{2}}{1+t_{c}^{2}}$, in (2.22), (2.23) and (2.24) we get a system of three rational equations with respect to $t_{a}, t_{b}$ and $t_{c}$. By solving the first derived equation of second degree with respect to $t_{a}$, we may obtain two solutions $t_{a_{1}}=$ $f_{1}\left(t_{b}\right)$ and $t_{a_{2}}=f_{2}\left(t_{b}\right)$. Similarly, by solving the second derived equation of second degree with respect to $t_{c}$, we may obtain two solutions $t_{c_{1}}=$ $f_{1}\left(t_{b}\right)$ and $t_{c_{2}}=f_{2}\left(t_{b}\right)$.

By replacing these pairs of solutions with respect to $\left(t_{a}\left(t_{b}\right), t_{c}\left(t_{b}\right)\right)$ in the third derived equation we obtain a rational equation which depend only on $t_{b}$.

The author is grateful to Professor Dr. Vassilios Papageorgiou for many fruitful discussions and for his comments on this particular problem.

\section{REFERENCES}

[1] V. Boltyanski, H. Martini, V. Soltan, Geometric Methods and Optimization Problems, Kluwer, Dordrecht-Boston-London, 1999.

[2] A. Cotsiolis and A. Zachos, Corrigendum to "The weighted Fermat-Torricelli problem on a surface and an "inverse" problem", J. Math. Anal. Appl., 376, no. 2 (2011) 760.

[3] E.J. Cockayne, On the Steiner problem, Canad. Math. Bull. 10 (1967), 431-450. 
[4] E.J. Cockayne, On Fermat's problem on the surface of a sphere, Math. Mag. 45 (1972), 216-219.

[5] J. Dolan, R. Weiss, J. MacGregor Smith, Minimal length tree networks on the unit sphere. Topological network design (Copenhagen, 1989). Ann. Oper. Res. 33 (1991), no. 1-4, 503535.

[6] P. Fletcher, S. Venkatasubramanian, S. Joshi, The geometric median on Riemannian manifolds with application to robust atlas estimation, NeuroImage $\mathbf{4 5}$ (2009) S143-S152.

[7] A.O. Ivanov and A.A. Tuzhilin, Geometry of minimal nets and the onedimensional Plateau problem, Russian Math. Surveys 47 (1992), no. 2, 59-131.

[8] S. Naya and N. Innami A comparison theorem for Steiner minimum trees in surfaces with curvature bounded below. Tohoku Math. J. (2) 65 (2013), no. 1, 131157.

[9] J.F. Weng, Steiner trees on curved surfaces, Graphs Combin. 17 (2001), no. 2, 353363.

[10] A.N. Zachos and G. Zouzoulas, The weighted Fermat-Torricelli problem and an "inverse" problem, J. Convex Anal,, 15, no. 1 (2008) 55-62.

[11] A. Zachos and A. Cotsiolis, The weighted Fermat-Torricelli problem on a surface and an "inverse" problem, J. Math. Anal. Appl., 373, no. 1 (2011) 44-58.

[12] A.N. Zachos, Location of the weighted Fermat-Torricelli point on the K-plane, Analysis (Munich), (2013), 33, 1001-1006.

[13] A.N. Zachos, A plasticity principle of convex quadrilaterals on a convex surface of bounded specific curvature, Acta Appl. Math. (2014),129, no. 1, 81-134.

University of Patras, Department of Mathematics, GR-26500 Rion, GREECE

E-mail address: azachos@gmail.com 